\title{
Diagnostic Value of Urinary Dysmorphic Erythrocytes in Clinical Practice
}

\author{
Meindert J. Crop ${ }^{a}$ Yolanda B. de Rijke ${ }^{b}$ Paul C.M.S. Verhagen ${ }^{c}$ \\ Karlien Cransberg $^{d}$ Robert Zietse $^{a}$ \\ Departments of ${ }^{\mathrm{a}}$ Internal Medicine, ${ }^{\mathrm{b}} \mathrm{Clinical}$ Chemistry, ${ }^{\mathrm{C}}$ Urology and ${ }^{\mathrm{d}}$ Pediatrics, Erasmus Medical Center, \\ Rotterdam, The Netherlands
}

\section{Key Words}

Diagnostic value $\cdot$ Urinary dysmorphic erythrocytes

Hematuria • Erythrocyturia · Red blood cells

\begin{abstract}
Background: In clinical practice, discriminating between glomerular and nonglomerular causes of hematuria is often difficult. Dysmorphic red blood cells (dRBC) in the urinary sediment are claimed to be effective, but the cutoff points in the literature vary. This follow-up study aimed to determine the diagnostic value of dRBC. Methods: We investigated 134 hematuria patients in the departments of nephrology and urology. To diagnose the origin of hematuria, urological and/or nephrological examination was performed and the \%dRBC identified by microscopy. Follow-up was performed after 3.5 years. Results: The cause of hematuria was proven in 68 patients (35\% glomerular; $65 \%$ nonglomerular). Patients with glomerular disease had significantly more albuminuria and $\mathrm{dRBC}$ than patients with nonglomerular disease, but the \%dRBC ranged from 1 to $50 \%$ and no optimal cutoff could be identified. Logistic regression analysis showed that \%dRBC had a predicted probability to diagnose glomerular disease of $77.9 \%$ (area under the curve, AUC, 0.85). When \%dRBC was combined with other risk factors such as serum creatinine, sex, age, dipstick erythrocyte
\end{abstract}

or proteinuria score and number of casts, the predictive probability increased to $90.6 \%$ (AUC 0.97). Follow-up of the included patients showed no benefit of dRBC to identify patients at risk for glomerular disease. Conclusions: The diagnostic value of routinely collected urinary $\mathrm{dRBC}$ to diagnose glomerular disease in patients presenting with hematuria is modest. However, including dRBC with other variables, such as age and erythrocyte score on dipstick testing may increase the sensitivity, but needs to be confirmed in another, preferably larger, population.

Copyright $\odot 2010$ S. Karger AG, Basel

\section{Introduction}

Hematuria is a common problem in clinical practice, with a reported prevalence ranging from 0.2 to $16.1 \%$, depending on population screened [1-2]. Transient hematuria can be caused by vigorous exercise, sexual intercourse, mild trauma or menstrual contamination. Persistent hematuria can generally be divided into hematuria of glomerular and nonglomerular sources. Common causes of glomerular hematuria are glomerulonephritis, IgA nephropathy, thin membrane disease and Alport's disease [3]. Hematuria of nonglomerular origin usually results from urological abnormalities,

\section{KARGER}

Fax +4161306 1234 E-Mail karger@karger.ch www.karger.com
(C) 2010 S. Karger AG, Basel

$1660-2110 / 10 / 1153-0203 \$ 26.00 / 0$

Accessible online at:

www.karger.com/nec
Robert Zietse, MD

Dr. Molewaterplein 50

PO Box 2040

NL-3000 CA Rotterdam (The Netherlands)

Tel. +31 10704 0704, Fax +31 10436 6372,E-Mail r.zietse@ erasmusmc.nl 
such as urinary tract infections, urological malignancy or urolithiasis.

As it is important to exclude the presence of a urological malignancy as the source of bleeding, patients presenting with hematuria primarily undergo urological analysis [4-5]. According to the guidelines of the American Urological Association, the urological evaluation of hematuria should include radiological imaging of the upper urinary tract followed by cystoscopic evaluation of the urinary bladder or additional examinations, depending on additional risk factors for urological disease [5]. Patients with persistent hematuria in whom the urological analysis is negative and having additional symptoms such as hypertension, proteinuria or renal impairment, should be referred to the nephrologist to be evaluated for glomerular disease.

Urinalysis is still considered an important diagnostic tool for nephrologists. Although dipstick testing is easy to perform in general practice, the morphology of urinary red blood cells (RBC) has been advocated as a fine diagnostic tool to differentiate between glomerular and nonglomerular causes of hematuria [6-8]. In glomerular hematuria, the variation in size and shape of the urinary $\mathrm{RBC}$ is increased, and such $\mathrm{RBC}$ are generally called dysmorphic RBC (dRBC). The exact pathophysiological mechanism of the formation of $\mathrm{dRBC}$ is unknown. Nevertheless, it is hypothesized, based on in vitro evidence, that $\mathrm{dRBC}$ are $\mathrm{RBC}$ which leaked through the diseased glomerulus and were damaged by mechanical and osmotic influences during their passage through the tubular system of the kidney, especially the collecting duct [9-13]. In contrast, in nonglomerular hematuria urinary $\mathrm{RBC}$ have a more uniform morphology and are therefore called isomorphic RBC.

In theory, a low percentage of $\mathrm{dRBC}(\% \mathrm{dRBC})$ excludes a glomerular cause of hematuria and it is therefore not indicated to refer these patients to a nephrologist, whereas a higher \%dRBC may demand further examination for glomerular disease. However, in the literature there is widespread controversy regarding the diagnostic value of urinary $\mathrm{dRBC}$ to identify the hematuria as glomerular in origin, because the criteria for dysmorphism of the urinary erythrocytes are vague and not standardized. Various dRBC have been identified based on their morphology such as acanthocytes or G1 cells [14-17]. Moreover, reported cutoff points of the \%dRBC range from 10 to $90 \%$, depending on screened population and study design [6, 18-20].

The present study was undertaken to determine the diagnostic value of urinary $\mathrm{dRBC}$ in 134 patients present- ing with hematuria in both urological and nephrological outpatient departments. After inclusion, the percentage of urinary $\mathrm{dRBC}$ was determined and was correlated with the clinical diagnosis of the source of the hematuria.

\section{Patients and Methods}

\section{Study Protocol}

Clinicians (both nephrologists and urologists) included patients referred for hematuria to the outpatient departments of nephrology and urology in the Erasmus Medical Center (20022004). Hematuria was defined as $1+(\geq 20 \mathrm{RBC} / \mu \mathrm{l})$ after dipstick. During the study period, the treating physicians remained responsible for the care of the patients included in this study. Treating physicians were not aware of the results of urinalysis and the investigators did not intervene at any point. After a follow-up of $3.8(0-6.7)$ years, it was investigated whether the included hematuria patients had developed glomerular disease. Follow-up information was retrieved from the hospital electronic information system.

\section{Study Groups}

Per patient, the final diagnosis was collected by reviewing clinical charts, the electronic hospital information system and discharge letters. The level of certainty that the definitive diagnosis was indeed the cause of hematuria was scored in five diagnostic groups (numbered -2 to +2 ; table 1). Patients in whom a glomerular source of hematuria was proven by renal biopsy (e.g. glomerulonephritis or IgA nephropathy) were classified as +2 , whereas those in whom urological examination identified a definitive urological source of bleeding (e.g. urinary tract infection, malignancy of the urinary tract or urolithiasis) were classified as -2 . Cases in which the cause of hematuria was totally unknown were coded as 0 . In diagnostic groups +1 and -1 , there were findings indicating either a glomerular or a urological source, but no final diagnosis for the hematuria was made. In our analyses of the predictive value of urinary $\mathrm{dRBC}$, only patients with a proven cause (i.e. diagnostic groups -2 and +2 ) were included and the percentage of urinary $\mathrm{dRBC}$ correlated with clinical findings and definitive diagnosis.

\section{Analytical Methods}

Fresh urine samples were analyzed within $2 \mathrm{~h}$ after voiding or fixated with CellFIX ${ }^{\mathrm{TM}}$ (Becton Dickinson, San Jose, Calif., USA). Firstly, before microscopic examination of the urine sediment, the urine specimens were routinely examined with an automated semiquantitative urinalysis using Combur ${ }^{10}$ Test $M$ strips on a Miditron ${ }^{\circledR} \mathrm{M}$ (Roche Diagnostics, Mannheim, Germany). Hematuria was scored in,+++ and +++ corresponding to 3-20, 20-100 and $>100$ erythrocytes/ $\mu l$, respectively. Using the same test system, albuminuria was also measured semiquantitatively $(+=$ 200-500 mg/l; ++ = 500-1,000 mg/l; +++ = >1,000 mg/l) [21]. Urinary $\mathrm{pH}$ was measured on a semiquantitative scale with increments of 0.5 . To estimate renal function of each patient, serum creatinine was measured.

Next, urine samples (6-12 ml, depending on concentration of erythrocytes) were centrifuged in a Kova tube (Instruchemie, Delfzijl, The Netherlands) at 1,600 rpm for $5 \mathrm{~min}$. Supernatant was 
Table 1. Diagnostic groups

\begin{tabular}{lll}
$\begin{array}{l}\text { Diagnostic } \\
\text { group }\end{array}$ & $\begin{array}{l}\text { Diagnostic cause of } \\
\text { hematuria }\end{array}$ & Final diagnoses \\
\hline-2 & Proven urological (44) & $\begin{array}{l}\text { Urinary tract infection (21) } \\
\text { Obstructive uropathy (10), including: } \\
\text { urolithiasis (5) } \\
\text { benign prostate hypertrophy (1) } \\
\text { malignancy of the urinary tract (4) }\end{array}$ \\
& & $\begin{array}{c}\text { Other (10): e.g. trauma, (congenital) anatomic abnormalities, } \\
\text { anticoagulant drugs } \\
\text { Non-glomerular renal disease (3): e.g. fibrosis }\end{array}$ \\
& & \\
-1 & Possible urological (14) & \\
0 & Uncertain (35) & \\
+1 & Possible glomerular (16) & Glomerulonephritis (18): primary/secondary \\
& & IgA nephropathy (6)
\end{tabular}

Figures in parentheses indicate number of cases.

discarded, and a drop of sediment suspension was placed on a slide with a covering glass. The presence of erythrocyte casts was examined using a bright-field microscope (Olympus, PAES, The Netherlands), while the presence of $\mathrm{dRBC}$ was examined using phasecontrast microscopy under high-power magnification $(\times 400)$. In each case, 100 erythrocytes were counted to determine the percentage of dysmorphic erythrocytes. Dysmorphic erythrocytes were defined using the criteria as reported previously [6, 22-23]. In brief, dysmorphic erythrocytes exhibited irregular membranes or small surface blebs and showed an annular or vesicular structure. Hematuria of a glomerular source shows a polymorphic aspect and erythrocyte casts can be present. In this study, we did not discriminate between the different types of dysmorphic erythrocytes, although the presence of erythrocyte casts was scored separately.

Examination was carried out by two well-trained technicians. If trained technicians were not present at the time the urine sample was delivered to the laboratory, a CellFIX fixation method was used as described by Huussen et al. [24]. The technicians were blinded to their colleagues' results and the patients' clinical information.

\section{Statistical Analysis}

The level of albuminuria and the \% $\mathrm{dRBC}$ were compared using the Fisher exact test. The mean \%dRBC in the five diagnostic groups were compared using a one-way ANOVA. Serum creatinine levels in these groups were compared using Friedman's ANOVA by ranks. If either yielded a significant $F$, multiple posthoc comparisons were performed using the Student-Newman-Keuls test. Interobserver variation of counted \%dRBC was assessed by means of a Bland-Altman plot and the calculation of the Wilcoxon signed ranks test. Sensitivity was calculated as the proportion of all patients with the disease (true positives + false negatives) who indeed have a positive test result (true positives), as defined previously [25]. Specificity was calculated as proportion of all patients without the disease and a negative test result (true negatives) of all those without the disease (true negatives + false positives). Binary logistic regression analysis was performed to make a prediction model for the variables: (1) dipstick erythrocytes score, (2) \%dRBC; (3) dipstick proteinuria score; (4) sex; (5) age; (6) number of erythrocyte casts, and (7) serum creatinine. ROC curves of the predicted probabilities were made. Data were analyzed using SPSS (version 15.0; Chicago, Ill., USA). Data are expressed as mean \pm standard deviation, or as median (range), depending on the distribution of the data. Area under the curve (AUC) is shown with $95 \%$ confidence interval. Statistical significance was defined as $\mathrm{p}<0.05$.

\section{Results}

\section{Patients}

In this study, 134 patients presenting with hematuria in the outpatient departments of nephrology and urology were included. In total, 93 adults and 41 children $(<18$ years) were included, including 68 females (50.7\%). The median age of this study population was 43 (1-86) years. The median creatinine concentration in serum was 71.5 $\mu \mathrm{M}(11-454)$.

\section{Semiquantitative Urinalysis}

Hematuria in the included patients had the following distribution: + in 24 (17.9\%), ++ in 19 (14.2\%) and +++ in 91 (67.9\%; table 2). Albuminuria was present in $26.9 \%$ (36/134) of all cases, and higher levels of albuminuria were significantly associated with higher levels of hematuria $(\mathrm{p}<0.05)$. Interestingly, in the group with proven uro- 


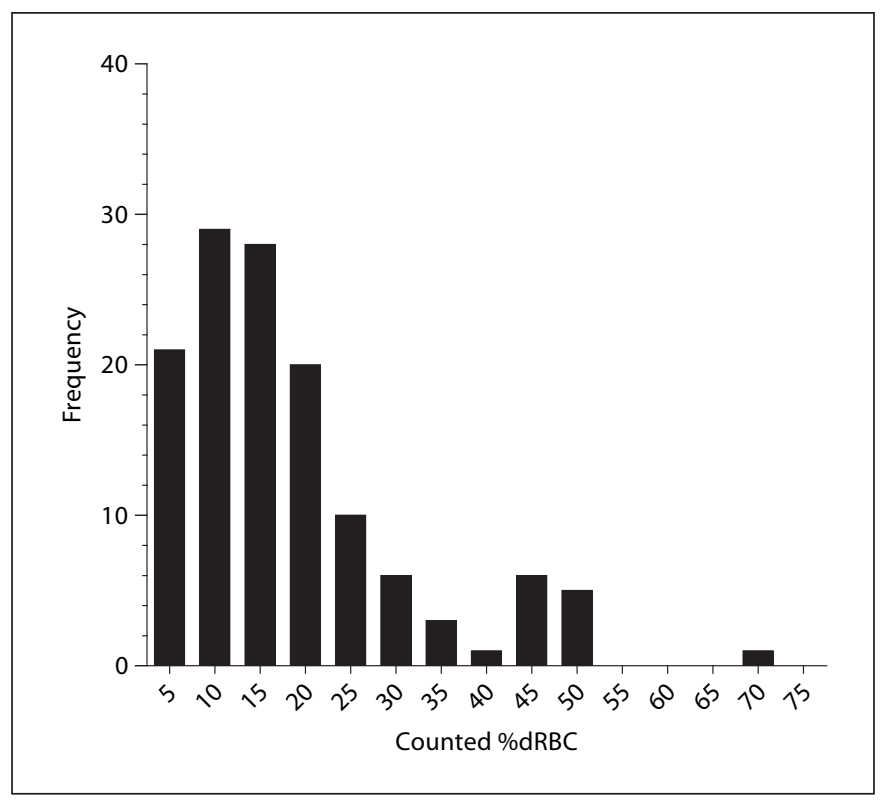

Fig. 1. Frequency distribution of microscopically counted $\% d R B C$ in 134 hematuria patients. Percentages are shown as medians of two measurements.

logical disease, i.e. diagnostic group -2 , there were still 6 patients who had $3+$ albuminuria scored on the test strip. However, the mean counted percentages of urinary dRBC in these patients ranged from 1 to 18 . The median urinary $\mathrm{pH}$ in the general study population was $6.0(5.0-8.0)$.

\section{Microscopic Analysis of the Urine Sediment}

The $\% \mathrm{dRBC}$ detected in the urine samples ranged from 1 to $71 \%$. Figure 1 shows the distribution of the percentages of urinary dRBC of all 134 hematuria patients included in this study population. There was no significant difference in \%dRBC counted by the two technicians. Figure 2 shows a Bland-Altman plot of the mean differences of the dRBC counts by observer 1 and 2 . The $\% \mathrm{dRBC}$ was significantly higher in the ++ than in the + and +++ hematuria groups $(\mathrm{p}<0.05$; table 2$)$. Erythrocyte casts were found in 6 of the 134 samples.

\section{Diagnostic Performance}

Dysmorphic RBC

In total, we found 68 (51\%) patients with a proven cause of hematuria, either urological or glomerular. Table 1 shows the definitive diagnoses of the patients with a proven origin of the hematuria after urological and/or nephrological examination. In $66 \%(27 / 41)$ of the pediatric pa-

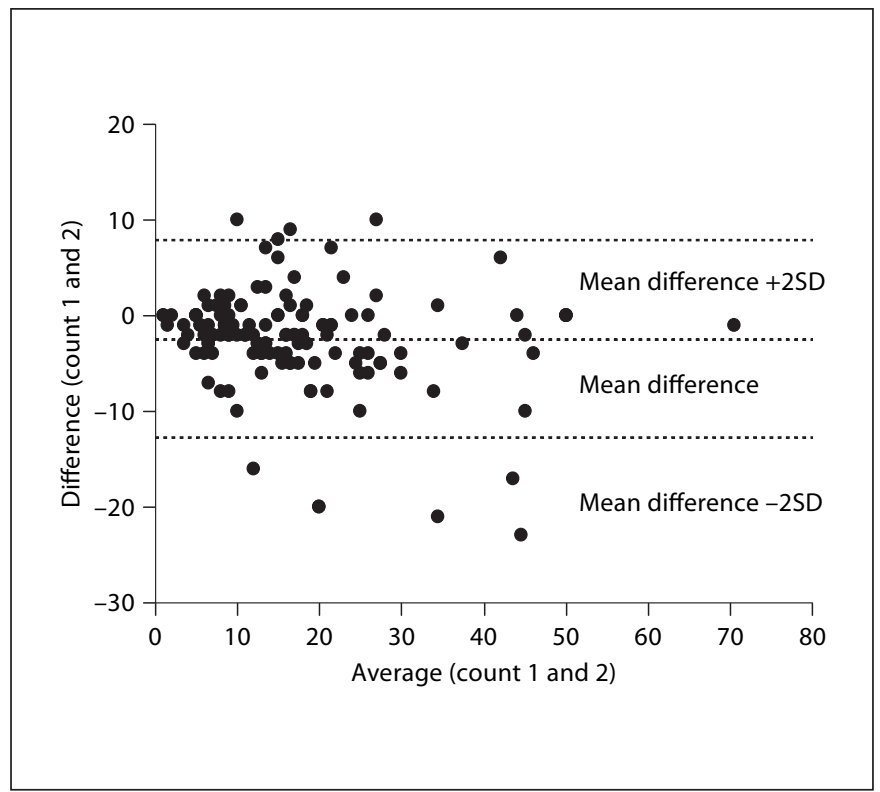

Fig. 2. Interobserver variability of counted \%dRBC (Bland-Altman plot). The mean differences between the counted percentages of urinary $\mathrm{dRBC}$ by 2 technicians (count 1 and count 2) were plotted.

Table 2. Results of urine semiquantitative test strip and microscopic analysis of 134 hematuria patients

\begin{tabular}{lllll}
\hline & \multicolumn{3}{l}{ Hematuria } & \\
\cline { 2 - 5 } & $\begin{array}{l}\text { overall } \\
(\mathrm{n}=134)\end{array}$ & $\begin{array}{l}+ \\
(\mathrm{n}=24)\end{array}$ & $\begin{array}{l}++ \\
(\mathrm{n}=19)\end{array}$ & $\begin{array}{l}+++ \\
(\mathrm{n}=91)\end{array}$ \\
\hline $\begin{array}{llll}\text { Albuminuria } \\
+\end{array}$ & & & & \\
++ & & 0 & 2 & 10 \\
+++ & 0 & 2 & 9 \\
$\quad \begin{array}{l}\text { Total } \\
\text { \%dRBC }\end{array}$ & 36 & 1 & 1 & 11 \\
Erythrocyte casts & 6 & 1 & 5 & $30^{\mathrm{a}}$ \\
& & $15(10)$ & $26(12)^{\mathrm{a}, \mathrm{b}}$ & $17(13)$ \\
\end{tabular}

${ }^{\mathrm{a}} \mathrm{p}<0.05$ vs. $+{ }^{\mathrm{b}} \mathrm{p}<0.05$ vs. +++ . Values for $\% \mathrm{dRBC}$ are expressed as mean (SD).

tients, a proven cause of hematuria was found: $48 \%(13 / 27)$ nonglomerular and 52\% (14/27) glomerular. In adult patients, 79\% (33/42) had a urological and 21\% (9/42) had glomerular pathology as the cause of hematuria.

The percentage of urinary $\mathrm{dRBC}$ differed significantly between the five diagnostic groups and was highest in the group with proven glomerular disease $(p<0.05$; table 3$)$. 
Table 3. Characteristics and results of urinalysis of diagnostic groups

\begin{tabular}{|c|c|c|c|c|c|}
\hline Diagnostic group & $\begin{array}{l}-2 \\
\text { Proven } \\
\text { urological }\end{array}$ & $\begin{array}{l}-1 \\
\text { Possible } \\
\text { urological }\end{array}$ & $\begin{array}{l}0 \\
\text { Uncertain }\end{array}$ & $\begin{array}{l}1 \\
\text { Possible } \\
\text { glomerular }\end{array}$ & $\begin{array}{l}2 \\
\text { Proven } \\
\text { glomerular }\end{array}$ \\
\hline Patients & 44 & 15 & 35 & 16 & 24 \\
\hline Female sex & $20(45.5 \%)$ & $11(73.3 \%)$ & $19(54.3 \%)$ & $5(31.3 \%)$ & $9(37.5 \%)$ \\
\hline Age & $41.9 \pm 24.4$ & $45.9 \pm 27.8$ & $48.5 \pm 17.4$ & $31.6 \pm 20.1^{\mathrm{c}}$ & $21.8 \pm 15.4^{\mathrm{a}-\mathrm{c}}$ \\
\hline Children & $13(28.3 \%)$ & $4(28.6 \%)$ & $3(8.6 \%)$ & $7(43.8 \%)$ & $14(60.9 \%)$ \\
\hline Serum creatinine, $\mu \mathrm{M}$ & $79(12-454)$ & $59(32-273)$ & $74(27-297)$ & $71(11-110)$ & $64(29-211)$ \\
\hline \multicolumn{6}{|c|}{ Semiquantitative urinalysis } \\
\hline \multicolumn{6}{|l|}{ Albuminuria } \\
\hline $1+$ & 4 & 1 & 0 & 0 & 7 \\
\hline $2+$ & 5 & 0 & 1 & 2 & 3 \\
\hline $3+$ & 6 & 1 & 0 & 1 & 5 \\
\hline Total & $15 / 46$ & $2 / 14$ & $1 / 35$ & $3 / 16$ & $15 / 23^{\mathrm{a}-\mathrm{d}}$ \\
\hline \multicolumn{6}{|l|}{ Erythrocyturia } \\
\hline $1+$ & 9 & 5 & 9 & 0 & 1 \\
\hline $2+$ & 7 & 2 & 5 & 4 & 1 \\
\hline $3+$ & 30 & 7 & 21 & 12 & 21 \\
\hline Total & 46 & 14 & 35 & 16 & 23 \\
\hline $\mathrm{pH}$ & $6.0(5.0-8.0)$ & $6.0(5.0-8.0)$ & $6.0(5.0-8.0)$ & $6.0(5.0-7.0)$ & $6.0(5.0-8.0)$ \\
\hline \multicolumn{6}{|l|}{ Microscopic analysis } \\
\hline$\% \mathrm{dRBC}$ & $12 \pm 7$ & $21 \pm 13$ & $18 \pm 15$ & $22 \pm 13^{\mathrm{a}}$ & $25 \pm 13^{\mathrm{a}, \mathrm{c}}$ \\
\hline Erythrocyte casts & 0 & 1 & 2 & 0 & 3 \\
\hline
\end{tabular}

Table 4. Sensitivity and specificity of $\% \mathrm{dRBC}$ for diagnosing glomerular disease at various cutoff points in 68 patients with proven disease

\begin{tabular}{rllrr}
\hline Cutoff points & $\begin{array}{l}\text { Positive test for proven } \\
\text { urological disease, } n\end{array}$ & $\begin{array}{l}\text { Positive test for proven } \\
\text { glomerular disease, } n\end{array}$ & $\begin{array}{l}\text { Sensitivity of \%dRBC } \\
\text { for glomerular disease, \% }\end{array}$ & $\begin{array}{l}\text { Specificity of \%dRBC } \\
\text { for glomerular disease, \% }\end{array}$ \\
\hline $0 \%$ & 44 & 24 & $100(24 / 24)$ & $0(44 / 44)$ \\
$10 \%$ & 24 & 23 & $96(23 / 24)$ & $45(20 / 44)$ \\
$20 \%$ & 4 & 13 & $54(13 / 24)$ & $91(40 / 44)$ \\
$30 \%$ & 1 & 6 & $25(6 / 24)$ & $98(43 / 44)$ \\
$40 \%$ & 0 & 5 & $21(5 / 24)$ & $100(44 / 44)$ \\
$50 \%$ & 0 & 2 & $8(2 / 24)$ & $100(44 / 44)$ \\
\hline
\end{tabular}

Albuminuria was also significantly higher in the group in which the hematuria resulted from a glomerular disease $(p<0.05)$. Serum creatinine concentrations were not significantly different in the various diagnostic groups. Patients with proven glomerular hematuria were significantly younger than patients in the other diagnostic groups $(\mathrm{p}<0.05)$.
Cutoff Value

As various cutoff points have been reported in the literature, we were interested what the effect was of different cutoff values on the sensitivity and specificity of urinary dRBC for glomerular disease. In table 4, the results of these calculations are shown. Using a cutoff point of $40 \%$ dRBC showed that none of the patients with a

Nephron Clin Pract 2010;115:c203-c212 
Fig. 3. \%dRBC per diagnostic group defined on certainty of the cause of hematuria. At a percentage of $40 \% \mathrm{dRBC}$, a horizontal line is drawn showing the diagnostic value of $\mathrm{dRBC}$ at this cutoff value for each diagnostic group: all patients with proven urological pathology had less than $40 \%$ dRBC, whereas 5 patients with proven glomerular pathology had more than $40 \%$. In 3 diagnostic groups (possible urological, uncertain and possible glomerular), no final cause of hematuria could be made.

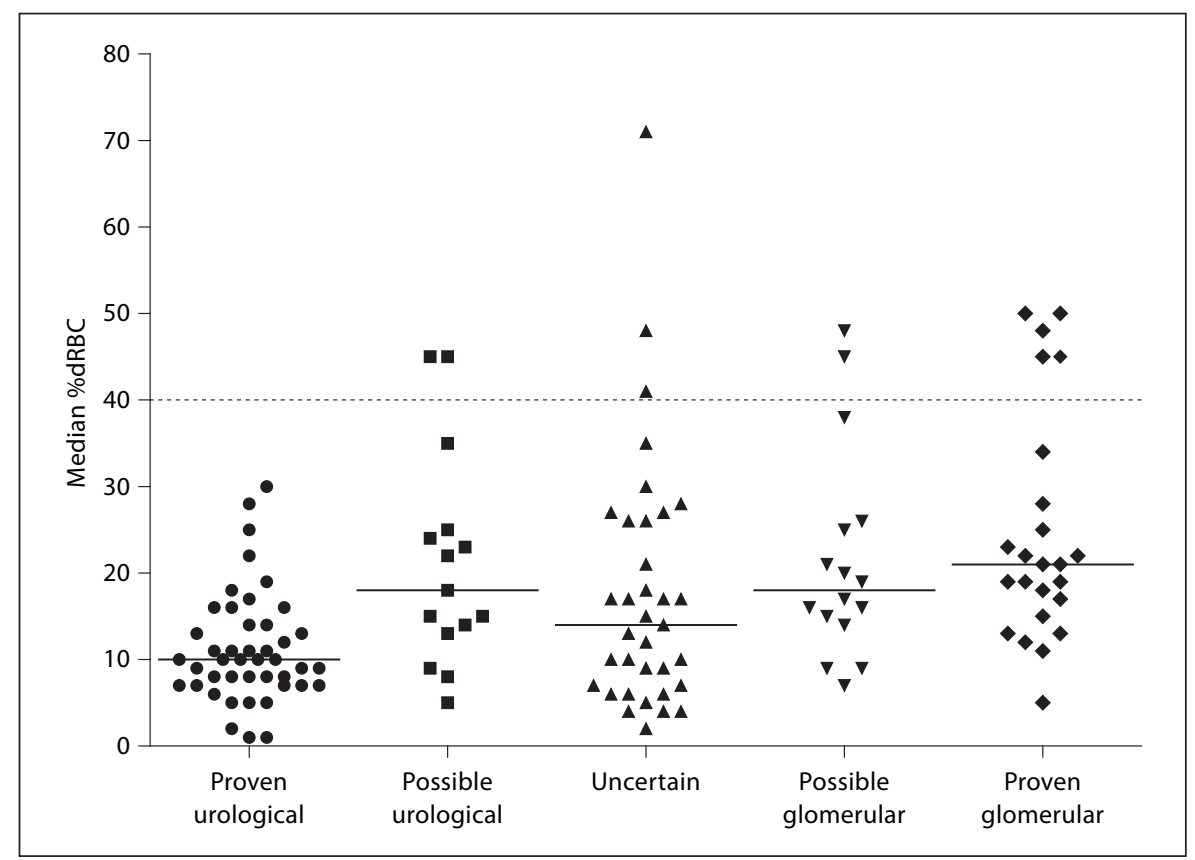

proven urological cause of hematuria had an increased $\% \mathrm{dRBC}$ in their urine (fig. 3), while only 5 of the 23 patients with a histological proven glomerular cause of hematuria had an elevated percentage $(>40 \%)$ of urinary dRBC. Thus, at a $40 \%$ cutoff point the sensitivity of urinary $\mathrm{dRBC}$ for excluding glomerular disease in patients with urological diseases was $100 \%(46 / 46 \mathrm{dRBC}<40 \%)$, while still $78 \%$ of the patients with a glomerular cause of hematuria had less than $40 \% \mathrm{dRBC}$. None of the patients with proven urological disease showed $\mathrm{ARBC}$ above the cutoff of $40 \%$.

Binary logistic regression analysis of all patients with proven causes of hematuria showed that the predicted probability of \%dRBC for glomerular disease was $77.9 \%$. The ROC curve of the predicted probability of $\% \mathrm{dRBC}$ is shown in figure $4 \mathrm{a}$ with an estimated AUC of 0.84 (0.76$0.95)$. Addition of proteinuria to $\% \mathrm{dRBC}$ hardly increased the predicted probability to $79.4 \%$, AUC $0.86(0.77-0.95$; fig. 4a). The predicted probability for glomerular disease improved to $90.6 \%$, AUC 0.97 (0.94-1.00) when the following variables were added to the prediction model: (1) dipstick erythrocyte score, (2) \%dRBC, (3) dipstick proteinuria score, (4) sex, (5) age, (6) erythrocyte casts and (7) serum creatinine (fig. 4b). The regression formula of this full model was: (glomerular disease $)=-14.9-3.93$. (erythrocyte score) $+0.48 \cdot(\% \mathrm{dRBC})+0.35 \cdot$ (proteinuria score) $-2.29 \cdot($ sex $)-0.10 \cdot$ (age) $+21.54 \cdot$ (erythrocyte casts) $-0.004 \cdot($ serum creatinine).
Based on our data, only \%dRBC, dipstick erythrocyte score and age significantly contributed to the prediction model. The regression formula of this reduced model was: $($ glomerular disease $)=-8.7+0.25 \cdot(\% \mathrm{dRBC})+2.13$ - (erythrocyte score) $-0.058 \cdot$ (age). Figure $4 \mathrm{~b}$ shows the ROC curve of the predicted probabilities of this reduced model. The predictive probability of this model was $85.3 \%$ with an AUC of 0.95 (0.89-1.00).

\section{Erythrocyte Casts}

In our study, erythrocyte casts were seen in 6 out of 134 cases (table 2,3 ). Five of those patients had ++ or more erythrocytes on the test strip. In 3 out of the 6 , the origin of hematuria (all glomerular) was found. One patient had IgA glomerulopathy and 2 patients suffered from glomerulonephritis. The percentages of urinary $\mathrm{dRBC}$ were 5,21 and 34 , respectively.

\section{Follow-Up}

To determine whether dysmorphic erythrocytes had a predictive value for the development of glomerular disease in the long-term, we reevaluated several years after inclusion whether the hematuria patients developed glomerular disease. The median follow-up time was approximately 3.8 years, with a maximum of 6.7 years. One year after inclusion, 29 patients were lost to follow-up, which was likely due to discharge or referral of patients to other hospitals. The follow-up of the rest of the patients was 1-4 




Fig. 4. ROC curves of predicted probabilities for glomerular disease in patients with proven disease. a ROC curves of the predicted probability of \%dRBC only, proteinuria only and a combination of \%dRBC and proteinuria to identify glomerular disease. b ROC curves of the predicted probability of \%dRBC only, all risk factors (full prediction model) and the reduced prediction model.

years (46 patients), while 59 patients had been followed for at least 4 years. It can be hypothesized that patients with a high percentage of urinary $\mathrm{dRBC}$ might be at risk to have or develop glomerular disease. From the 7 patients with more than $40 \% \mathrm{dRBC}$ and no previously diagnosed glomerular disease, one patient developed IgA nephropathy after a follow-up of 2.0 years. In contrast, there were 2 patients with a proven urological cause who both developed IgA nephropathy, while the $\% \mathrm{dRBC}$ was only 10 and 11 in their urine sediments.

\section{Discussion}

This study, performed in 134 patients presenting with hematuria in both urological and nephrological outpatient departments, shows that the measurement of urinary $\mathrm{dRBC}$ has a moderate diagnostic value to identify glomerular disease. Therefore, it may be useful in the urological department to identify patients with glomerular diseases who may benefit from referral to a nephrologist.

In only $51 \%$ of the patients in our study could a definite diagnosis explaining the hematuria be made after a

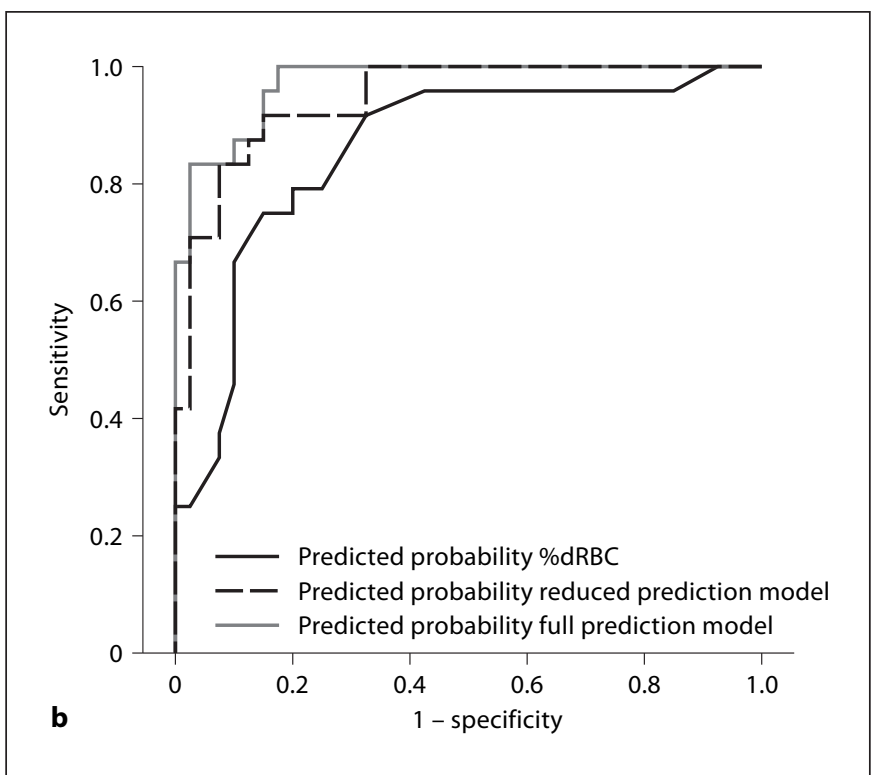

The full prediction model consisted of \%dRBC, erythrocyte score on dipstick, proteinuria score on dipstick, sex, age, serum creatinine and number of erythrocyte casts. The reduced prediction model consisted of \%dRBC, erythrocyte score on dipstick and age.

full urological and/or histopathological evaluation: 18\% with glomerular pathology versus $34 \%$ with urological pathology. Although this percentage appears low, previous studies reported similar percentages of identified causes of hematuria (ranging from 32 to $90 \%$ even after full examination) $[4,5,26]$. We failed to determine optimal diagnostic cutoff values, which was due to the wide frequency distribution of urinary $\mathrm{dRBC}$ in these patients.

\section{The Value of $d R B C$ in Urological Disease}

In our study, $34 \%$ of all patients had proven urological pathology. We found that these patients were significantly older than the patients in the other diagnostic groups. This most likely resulted from the fact that urinary tract infections, malignancies of the urinary tract, prostate hypertrophy and stones are more frequent in elderly $[27,28]$. All patients with a urological cause of hematuria had less than the arbitrary chosen level of $40 \% \mathrm{dRBC}$ in their urine. This would suggest that identifying $\geq 40 \% \mathrm{dRBC}$ in the urinary sediment excludes urological pathology. Using this argument, it has been suggested that all patients with more than $40 \%$ urinary dRBC should be referred to a nephrologist as they have a low risk of uro- 
logical disease and may be spared a full urological workup [26]. However, patients with a high \%dRBC due to subclinical glomerular abnormality (such as thin membrane disease) may also develop a urological malignancy. Indeed, the question remains how such high \%dRBC can occur in patients with a urological source of hematuria. There is no clear pathophysiological explanation for this, as $\mathrm{dRBC}$ are supposed to be formed in the tubular system of the kidney due to mechanical and osmotic changes and not in the lower urinary tract [29]. A possible explanation for the relatively high \%dRBC in these patients with an established urological cause could be the coexistence of (subclinical) glomerular disease pathology, such as thinmembrane disease. Some reports estimate the frequency of this disorder to be as high as $1-10 \%$ in the general population [30]. However, for obvious reasons, renal biopsy was not performed in these patients. Six patients with proven urological disease and low urinary $\mathrm{dRBC}(<40 \%)$ had a significant amount of albuminuria on the test strip. These patients would not have been identified by the use of only urinary $\mathrm{dRBC}$, but should nevertheless be referred to a nephrologist for the investigation of coexisting renal disease.

Follow-up showed that 2 patients with proven urological disease developed glomerular disease, although the $\% \mathrm{dRBC}$ in their urine sediment was only 10 and $11 \%$, respectively. This suggests that the determination of urinary $\mathrm{dRBC}$ is not useful to detect glomerular disease in the urological department, but may be useful to exclude glomerular disease in patients with urological diseases.

\section{The Value of $d R B C$ in Glomerular Disease}

In total, $18 \%$ of the patients enrolled in our study had biopsy-proven glomerular disease. When investigating the diagnostic value of urinary $\mathrm{dRBC}$ for glomerular disease, we found that the percentage of urinary $\mathrm{dRBC}$ was significantly higher in patients with proven glomerular disease than in patients with urological pathology. However, a low \%dRBC did not rule out glomerular pathology as the $\% \mathrm{dRBC}$ ranged from 1 to $50 \%$. Albuminuria is an important indicator of glomerular damage and was significantly more frequent in patients with proven glomerular hematuria. Two of these patients had no albuminuria at all, leaving $\mathrm{dRBC}$ as the only indicator of a glomerular abnormality. Conversely, 8 patients with proven glomerular disease had marked albuminuria but less than $40 \%$ urinary dRBC. These patients would not have been identified by the use of urinary $\mathrm{dRBC}$ alone, and this finding challenges the validity of $\mathrm{dRBC}$ as a diagnostic tool for glomerular disease. Serum creatinine, as an estimate of glomerular filtration rate, could also be of value for diagnosing glomerular disease. In our study, however, there was no significant difference in serum creatinine between the diagnostic groups. In the 5 patients with proven glomerular disease and $\mathrm{dRBC}>40 \%$, the median serum creatinine concentration was 61 (range 40-71) $\mu \mathrm{M}$ and did not add to the diagnosis. Another indication that glomerular pathology is the cause of hematuria is the presence of urinary erythrocyte casts which is virtually pathognomonic of glomerular bleeding. Unfortunately, they are a relatively insensitive marker and are frequently not present in urine of patients with glomerular pathology $[18,19]$. In our study, erythrocyte casts were observed in only 6 urine specimens, which were in 3 out of the 6 cases derived from patients with proven glomerular disease.

Furthermore, it can be hypothesized that using a combination of variables/risk factors such as \% dRBC, erythrocyte score on dipstick, proteinuria score on dipstick, sex, age, serum creatinine and number of erythrocyte casts, can increase the sensitivity for glomerular disease. In our hands, especially \%dRBC, age and erythrocyte score on dipstick showed a significant contribution to predict glomerular disease. Although these findings should first be confirmed in another, preferably larger, study population, we can speculate that the composition of a scoring system based on these risk factors may improve the identification of patients with glomerular disease. This can be of special interest in the department of urology to identify patients who need referral to a nephrologist.

\section{Study Limitations}

In our study, we did not routinely acidify hematuria patients before obtaining urine samples, and our analysis showed that the median $\mathrm{pH}$ of the urinary specimens was 6.0. This may have affected our results, as both the generation of $\mathrm{dRBC}$ and the formation of erythrocyte casts is enhanced by acidic urine $[9,29]$. Urinary osmolality has also been reported to be important for the formation of dRBC and erythrocyte casts [9]. However, in our study, urine osmolality was not measured and no specific effort was made to ensure that concentrated urine samples were obtained, although all samples were collected in the morning. All urine specimens were analyzed within $2 \mathrm{~h}$ after voiding or fixated with CellFIX. Moreover, we found that fixation did not affect the percentages of $\mathrm{ARBC}$ in the urine (data not shown), which is in line with the previous literature [24]. Possibly, the \%dRBC in patients with renal disease can be increased, thus increasing the sensitivity, 
by a more rigorous study protocol, controlling both $\mathrm{pH}$ and osmolality in the urine samples. However, in none of the previously reported studies was such a protocol applied. Moreover, preconditioning of the patient would severely hamper the application of this test in clinical practice.

To standardize measurements and to reduce the workload of conventional microscopic examination, automated systems using computer-assisted light microscopy [31, 32], immunocytochemical staining, flow cytometric analysis of erythrocytes [33] or measurement of size or mean corpuscular volume of erythrocytes [34] have been used in the laboratories. These methods, however, are still not qualified to detect and quantify urinary dRBC. The sensitivity and specificity of these methods to diagnose pathology are still under investigation, and manual microscopic examination is still required to identify some cell types [35]. The present study evaluated whether there is still reason to perform manual microscopic examination of urinary specimens in clinical practice.

An unanswered, yet important, question is: should all patients with isolated hematuria and high percentages of urinary $\mathrm{dRBC}$ be referred to a nephrologist? Follow-up of patients with more than $40 \% \mathrm{dRBC}$ and no previously proven glomerular disease showed that only one of the 7 developed glomerular disease during this period, suggesting that a high dysmorphism of urinary RBC does not predict glomerular disease in the short-term.

Furthermore, the definitive diagnosis of glomerular disease can only be made by renal biopsy. This is often not indicated, as the likelihood of finding a treatable disease in isolated glomerular hematuria is very low and these patients appear to have a low risk of progressive renal disease [36]. In these patients, a conservative policy is justified and regular monitoring of these patients for the development of hypertension, renal insufficiency and albuminuria appears sufficient.

In conclusion, this follow-up study in patients presenting with hematuria shows that the diagnostic value of routinely collected urinary $\mathrm{dRBC}$ to diagnose glomerular disease is modest, as the presence of a low \%dRBC failed to exclude glomerular disease. Although there is an urgent need for both urologists and nephrologists to discriminate between hematuria of glomerular and nonglomerular origin, we feel that currently the manual determination of urinary $\mathrm{dRBC}$ is not recommended in clinical practice. Making a scoring system based on a combination of several risk factors, especially \%dRBC, age and erythrocyturia, may increase the probability to identify glomerular disease. This needs to be further investigated in another, preferably larger, population.

\section{Acknowledgements}

The authors are indebted to the technicians of the Department of Clinical Chemistry for the analysis of all urine samples, and to the participating physicians in the Departments of Nephrology and Urology, including the Pediatric Departments for the inclusion of all hematuria patients.

\section{References}

1 Mohr DN, Offord KP, Owen RA, Melton LJ 3rd: Asymptomatic microhematuria and urologic disease. A population-based study. JAMA 1986;256:224-229.

$\checkmark 2$ Woolhandler S, Pels RJ, Bor DH, Himmelstein DU, Lawrence RS: Dipstick urinalysis screening of asymptomatic adults for urinary tract disorders. I. Hematuria and proteinuria. JAMA 1989;262:1214-1219.

- 3 Tomson C, Porter T: Asymptomatic microscopic or dipstick haematuria in adults: which investigations for which patients? A review of the evidence. BJU Int 2002;90:185198.

.

4 Sutton JM: Evaluation of hematuria in adults. JAMA 1990;263:2475-2480.

5 Grossfeld GD, Litwin MS, Wolf JS Jr, Hricak H, Shuler CL, Agerter DC, Carroll PR: Evaluation of asymptomatic microscopic hematuria in adults: The American Urological Association best practice policy - part II: patient evaluation, cytology, voided markers, imaging, cystoscopy, nephrology evaluation, and follow-up. Urology 2001;57:604610.

6 Fairley KF, Birch DF: Hematuria: a simple method for identifying glomerular bleeding. Kidney Int 1982;21:105-108.

7 Birch DF, Fairley KF, Whitworth JA, Forbes I, Fairley JK, Cheshire GR, Ryan GB: Urinary erythrocyte morphology in the diagnosis of glomerular hematuria. Clin Nephrol 1983; 20:78-84.
${ }_{8}$ Fogazzi GB, Edefonti A, Garigali G, Giani M, Zolin A, Raimondi S, Mihatsch MJ, Messa P: Urine erythrocyte morphology in patients with microscopic haematuria caused by a glomerulopathy. Pediatr Nephrol 2008;23: 1093-1100.

-9 Schramek P, Moritsch A, Haschkowitz H, Binder BR, Maier M: In vitro generation of dysmorphic erythrocytes. Kidney Int 1989; 36:72-77.

10 Tomita M, Kitamoto Y, Nakayama M, Sato T: A new morphological classification of urinary erythrocytes for differential diagnosis of glomerular hematuria. Clin Nephrol 1992; 37:84-89.

11 Rath B, Turner C, Hartley B, Chantler C: What makes red cells dysmorphic in glomerular haematuria? Pediatr Nephrol 1992;6: 424-427. 
-12 Georgopoulos M, Schuster FX, Porpaczy P, Schramek P: Evaluation of asymptomatic microscopic haematuria - influence and clinical relevance of osmolality and $\mathrm{pH}$ on urinary erythrocyte morphology. Br J Urol 1996;78:192-196.

13 Degrell P, Wagner Z, Szijarto IA, Wagner L, Marko L, Mohas M, Cseh J, Wittmann I: Morphology of glomerular hematuria is reproduced in vitro by carbonyl stress. Nephron Exp Nephrol 2008;110:e25-e30.

14 Kohler H, Wandel E, Brunck B: Acanthocyturia - a characteristic marker for glomerular bleeding. Kidney Int 1991;40:115-120.

$\checkmark 15$ Dinda AK, Saxena S, Guleria S, Tiwari SC, Dash SC, Srivastava RN, Singh C: Diagnosis of glomerular haematuria: role of dysmorphic red cell, G1 cell and bright-field microscopy. Scand J Clin Lab Invest 1997;57:203208

-16 Zaman Z, Proesmans W: Dysmorphic erythrocytes and G1 cells as markers of glomerular hematuria. Pediatr Nephrol 2000;14: 980-984.

-17 Nagahama D, Yoshiko K, Watanabe M, Morita Y, Iwatani Y, Matsuo S: A useful new classification of dysmorphic urinary erythrocytes. Clin Exp Nephrol 2005;9:304-309.

$\checkmark 18$ Fassett RG, Horgan BA, Mathew TH: Detection of glomerular bleeding by phase-contrast microscopy. Lancet 1982;1:1432-1434.

-19 Pollock C, Liu PL, Gyory AZ, Grigg R, Gallery ED, Caterson R, Ibels L, Mahony J, Waugh D: Dysmorphism of urinary red blood cells - value in diagnosis. Kidney Int 1989;36:1045-1049.

-20 Mohammad KS, Bdesha AS, Snell ME, Witherow RO, Coleman DV: Phase contrast microscopic examination of urinary erythrocytes to localise source of bleeding: an overlooked technique? J Clin Pathol 1993;46: 642-645.
21 Zeller A, Sigle JP, Battegay E, Martina B: Value of a standard urinary dipstick test for detecting microalbuminuria in patients with newly diagnosed hypertension. Swiss Med Wkly 2005;135:57-61

22 Roth S, Renner E, Rathert P: Microscopic hematuria: advances in identification of glomerular dysmorphic erythrocytes. J Urol 1991;146:680-684.

23 van der Snoek BE, Hoitsma AJ, van Weel C, Koene RA: Dysmorphic erythrocytes in urinary sediment in differentiating urological from nephrological causes of hematuria (in Dutch). Ned Tijdschr Geneeskd 1994;138: 721-726

24 Huussen J, Koene RA, Hilbrands LB: The (fixed) urinary sediment, a simple and useful diagnostic tool in patients with haematuria. Neth J Med 2004;62:4-9.

25 van Stralen KJ, Stel VS, Reitsma JB, Dekker FW, Zoccali C, Jager KJ: Diagnostic methods I: sensitivity, specificity, and other measures of accuracy. Kidney Int 2009;75:1257-1263.

26 Huussen J, Koene RA, Meuleman EJ, Hilbrands LB: Diagnostic approach in patients with asymptomatic haematuria: efficient or not? Int J Clin Pract 2006;60:557-561.

27 Khadra MH, Pickard RS, Charlton M, Powell $\mathrm{PH}$, Neal DE: A prospective analysis of 1,930 patients with hematuria to evaluate current diagnostic practice. J Urol 2000;163:524527.

28 Grossfeld GD, Litwin MS, Wolf JS, Hricak H, Shuler CL, Agerter DC, Carroll PR: Evaluation of asymptomatic microscopic hematuria in adults: The American Urological Association best practice policy - part I: definition, detection, prevalence, and etiology. Urology 2001;57:599-603.
29 Kitamoto Y, Yide C, Tomita M, Sato T: The mechanism of glomerular dysmorphic red cell formation in the kidney. Tohoku J Exp Med 1992;167:93-105.

30 Wang YY, Savige J: The epidemiology of thin basement membrane nephropathy. Semin Nephrol 2005;25:136-139.

31 Oktenli G, Tunca Y, Bulucu F, Vural A: Computer-assisted light microscopy in evaluating urinary red blood cell morphology. Clin Nephrol 1999;51:130-131.

-32 van den Broek D, Keularts IM, Wielders JP, Kraaijenhagen RJ: Benefits of the iq200 automated urine microscopy analyser in routine urinalysis. Clin Chem Lab Med 2008;46: 1635-1640.

33 Kore RN, Dow CS, Desai KM: A new automated system for urine analysis: a simple, cost-effective and reliable method for distinguishing between glomerular and nonglomerular sources of haematuria. BJU Int 1999;84:454-460.

34 Angulo JC, Lopez-Rubio M, Guil M, Herrero B, Burgaleta C, Sanchez-Chapado M: The value of comparative volumetric analysis of urinary and blood erythrocytes to localize the source of hematuria. J Urol 1999;162: 119-126.

35 Shayanfar N, Tobler U, von Eckardstein A, Bestmann L: Automated urinalysis: first experiences and a comparison between the Iris iQ200 urine microscopy system, the Sysmex UF-100 flow cytometer and manual microscopic particle counting. Clin Chem Lab Med 2007;45:1251-1256.

36 Nieuwhof C, Doorenbos C, Grave W, de Heer F, de Leeuw P, Zeppenfeldt E, van Breda Vriesman PJ: A prospective study of the natural history of idiopathic non-proteinuric hematuria. Kidney Int 1996;49:222-225. 